\title{
QUADRATIC MODELS FOR GENERIC LOCAL 3-PARAMETER BIFURCATIONS ON THE PLANE
}

\author{
FREDDY DUMORTIER AND PETER FIDDELAERS
}

\begin{abstract}
The first chapter deals with singularities occurring in quadratic planar vector fields. We make distinction between singularities which as a general system are of finite codimension and singularities which are of infinite codimension in the sense that they are nonisolated, or Hamiltonian, or integrable, or that they have an axis of symmetry after a linear coordinate change or that they can be approximated by centers. In the second chapter we provide quadratic models for all the known versal $k$-parameter unfoldings with $k=1,2,3$, except for the nilpotent focus which cannot occur as a quadratic system. We finally show that a certain type of elliptic points of codimension 4 does not have a quadratic versal unfolding.
\end{abstract}

\section{INTRODUCTION}

How complicated can local bifurcations be in quadratic systems, or in other terms, which phenomena can be described by germs of families of quadratic vector fields on the plane? We do not give a complete answer to this question, but we essentially treat two exemplary aspects. The first one concerns the singularities themselves, emphasizing their codimension as a quadratic system and as a general system. The second one concerns the relation between the quadratic unfolding of the singularity and its (versal) unfolding as a general system.

The complete treatment of the first problem is described in Chapter 1. We make a distinction between singularities having a finite codimension or having infinite codimension as a general system. The singularities of the first kind are listed completely, while those of the second kind are only checked to be nonisolated, or isolated but Hamiltonian, or integrable, or having an axis of symmetry after a linear coordinate change, or approachable by centers (these cases are of course not mutually distinct).

For the singularities of the first kind, the quadratic codimension reveals to be the same as the general one. It can reach at most 5 , and this only happens if the vector field is homogeneous. Different kinds of singularities of codimension 4 however occur.

Received by the editors December 1, 1989.

1980 Mathematics Subject Classification (1985 Revision). Primary 58FXX, 34CXX.

Key words and phrases. Quadratic planar vector fields, singularities, codimension, versal unfoldings, bifurcations. 
Since a purely linear singularity of the first kind is necessarily hyperbolic, we can restrict to nonhyperbolic singularities of degree exactly two. The semihyperbolic ones of the first kind have a codimension at most 3 . The same happens with the singularities having an infinitesimal rotation as 1-jet, as well as with the nilpotent singularities of cusp type. For all these singularities we can give in Chapter 2 an example of a quadratic family representing a versal unfolding (versal among general systems). As the bifurcation diagram of the cusp of codimension 3 is not algebraic (and even not analytic), we obtain a negative answer to a question raised by Coppel in [C] asking to characterize the phase portraits of quadratic systems by means of algebraic inequalities on the coefficients.

Nilpotent singularities of saddle or elliptic type occur in codimension 3 and 4. In Chapter 2 we give a quadratic example of a versal unfolding for a codimension 3 saddle and a codimension 3 elliptic point. In Chapter 3 , we show that the elliptic point of codimension 4 and type 1 does not have a quadratic versal unfolding. Essentially the reason is that the nilpotent focus of codimension 3 cannot be given a quadratic model. One can find a quadratic nilpotent saddlenode singularity of codimension 4 , but the remaining nilpotent singularities are of the second kind.

At last we show that among homogeneous quadratic vector fields the singularities have codimension 4 or 5 , before getting of the second kind. Of course such homogeneous quadratic singularities can also be considered to be of infinite codimension, because they are invariant under the transformation $(x, y, t) \mapsto(-x,-y,-t)$.

As we already mentioned, Chapter 2 contains quadratic models for all the known versal unfoldings-all the complete local bifurcations-of codimension at most 3, except for the nilpotent focus. In Chapter 3 is shown that in codimension 4 there exists a singularity for which it is definitely not possible to find a quadratic versal unfolding.

The proofs in this paper essentially consist of formal calculations-calculations of normal forms-which were performed on an Apollo 4000 using Macsyma, and using a program from Rand and Armbruster [R.A.]. Some of these calculations were also checked on a MacII using Mathematica. Concerning the normal forms which we use, as well as for the the proof of the versality of the unfoldings, we refer to [D1] or [D2, T1, T2, DRS1, DRS2, G.H.]. All the necessary definitions can also be found in these references.

\section{ChaPter 1. Singularities OF FINITE CODIMENSION}

Among the singularities which we encounter in differential equations

$$
\left\{\begin{array}{l}
\frac{d x}{d t}=P(x, y) \\
\frac{d y}{d t}=Q(x, y)
\end{array}\right.
$$

with $P$ and $Q$ polynomial of degree $\leq 2$, we will make a distinction between the singularities which are of finite codimension as a singularity of a general 
differential equation on the plane (see [T1] or [D1, 2] for a definition) and singularities which are of infinite codimension. The first will be called singularities of the first kind, while the latter will be said to be of the second kind. Singularities of the second kind will be detected by showing that they are either nonisolated, or that they are Hamiltonian, or that they have an axis of symmetry after a linear coordinate change, or that they are approachable by centers.

1.1. Hyperbolic singularities. These are of codimension 0 , as well among quadratic systems as among general systems. In both cases the hyperbolic singularities form an open and dense set. In the set of quadratic systems we work with the coefficient topology, while in the set of germs of singular $C^{\infty}$ vector fields we work with the usual topology as defined in [T1 and D1, 2], which is an inverse limit topology coming from the coefficient topologies of the jet spaces.

1.2. Semihyperbolic singularities. By this we mean singularities with 0 as a simple eigenvalue. We will show that in case of singularities of the first kind only semihyperbolic singularities of codimension $\leq 3$ occur, and the general as well as the quadratic codimensions are the same.

For this purpose we can suppose that the system is in canonical form (given by Jordan normal form theory):

$$
\left\{\begin{array}{l}
\frac{d x}{d t}=a x^{2}+b x y+c y^{2} \\
\frac{d y}{d t}=y+d x^{2}+e x y+f y^{2}
\end{array}\right.
$$

Since the center manifold is tangent to $E^{c}=\left\{(x, y) \in \mathbb{R}^{2} \mid y=0\right\}$ we can represent it as a (local) graph

$$
W^{c}=\left\{(x, y) \in \mathbb{R}^{2} \mid y=h(x)\right\} ;
$$

where $h: U \subset \mathbb{R} \rightarrow \mathbb{R}$ is defined on some neighborhood $U \subset \mathbb{R}$ of 0 , with $h(0)=h^{\prime}(0)=0$. The restriction to the center manifold is given by

$$
\frac{d x}{d t}=a x^{2}+b x h(x)+(h(x))^{2} .
$$

Now we calculate the Taylor series of $h(x)$ at $x=0$. Substituting $y=h(x)$ in the second component of (2), we obtain

$$
h^{\prime}(x)\left(a x^{2}+b x h(x)+c(h(x))^{2}\right)-\left(h(x)+d x^{2}+e x h(x)+f(h(x))^{2}\right)=0
$$

with conditions $h(0)=h^{\prime}(0)=0$. The solution of this equation can be approximated arbitrarily closely as a Taylor series at $x=0$. So we find

$$
h(x)=-d x^{2}+d(e-2 a) x^{3}-d\left(d f+e^{2}-5 a e-2 b d+6 a^{2}\right) x^{4}+O\left(x^{5}\right) .
$$

Substituting this Taylor approximation in (3), we obtain for the behaviour on the center manifold

$$
\begin{aligned}
\frac{d x}{d t}= & a x^{2}-b d x^{3}+\left(b d(e-2 a)+c d^{2}\right) x^{4} \\
& -\left(b d\left(d f+e^{2}-5 a e-2 b d+6 a^{2}\right)+2 c d^{2}(e-2 a)\right) x^{5}+O\left(x^{6}\right)
\end{aligned}
$$

From this it is readily seen that the next theorem holds. 
Theorem 1. The quadratic system (2) has:

(1) a saddle-node of codimension 1 if $a \neq 0$,

(2) a saddle or a node of codimension 2 if $a=0, b d \neq 0$,

(3) a saddle-node of codimension 3 if $a=0, b=0, c d \neq 0$.

If $a=0, b=0, c=0$ or if $a=0, d=0$ there is a curve of singularities so that the codimension is $\infty$.

The conclusion about the codimension as a quadratic system follows from the Tarski-Seidenberg theorem (appendix of [A.R.]).

1.3. Singularities with an infinitesimal rotation as 1-jet. Using the Jordan normal form theorem and multiplication with a positive number, we may suppose that these singularities have the form:

$$
\left\{\begin{array}{l}
\frac{d x}{d t}=-y+a x^{2}+b x y+c y^{2} \\
\frac{d y}{d t}=x+d x^{2}+e x y+f y^{2}
\end{array}\right.
$$

As has been shown by Bautin in [B], these singularities can be of codimension 1, 2 or 3 and in that case the codimension is the same among quadratic systems, or they are centers and are integrable. An account of these results can for instance be found in [Y]. Recently D. Schlomiuk [S] also wrote some nice account on this result, pointing out some mistakes in different other references and emphasizing some geometrical features of these centers. The formal normal form for these singularities in polar coordinates is

$$
\left(1+\sum_{i=1}^{\infty} a_{i} r^{2 i}\right) \frac{\partial}{\partial \theta}+\left(\sum_{j=1}^{\infty} b_{j} r^{2 j}\right) r \frac{\partial}{\partial r}
$$

and in case this represents a center all $b_{j}$ are zero, which is clearly a situation of infinite codimension.

1.4. Nilpotent singularities. Again using the Jordan normal form theorem, we can suppose that the system is in the canonical form:

$$
\left\{\begin{array}{l}
\frac{d x}{d t}=y+a x^{2}+b x y+c y^{2} \\
\frac{d y}{d t}=d x^{2}+e x y+f y^{2}
\end{array}\right.
$$

Up to a linear conjugacy we may even assume $d$ to be 0 or 1 .

First we treat the case $d=1$

Theorem 2. When $d=1$, system (6) has at the origin:

(1) a cusp singularity of codimension 2 if $e+2 a \neq 0$,

(2) a cusp singularity of codimension 3 if $e+2 a=0, b+2 f \neq 0$, $c \neq a\left(f-b-2 a^{2}\right)$.

Under all further restrictions, the cusp singularity is Hamiltonian or is symmetric with respect to an axis after a linear change of coordinates, and hence of $\infty$ codimension. 
Proof. Using the Takens normal form theorem [T1], we can formally give system (6) the following expression:

$$
\left\{\begin{array}{l}
\frac{d u}{d t}=v \\
\frac{d v}{d t}=\sum_{n \geq 2} a_{n} u^{n}+v \sum_{n \geq 2} b_{n} u^{n-1}
\end{array}\right.
$$

where $(u, v)$ are related to $(x, y)$ by a near-identity transformation (in the terminology of [G.H.]). Using Macsyma one easily finds the family of such transformations which perform these calculations up to terms of order 4 . The family is:

$$
\begin{aligned}
x= & r_{6} v^{4}+\left(\left(2 r_{3}+r_{1}^{2}\right) c+\left(r_{4}+r_{1} r_{2}\right) b+r_{2}^{2} a+r_{5}\right) u v^{3}+r_{4} v^{3} \\
& +\left(\left(6 r_{1} c+r_{2} b+2 r_{3}+r_{1}^{2}\right) f+\left(2 r_{2} c-2 r_{4}+r_{1} r_{2}\right) e\right. \\
& \left.+\left(3 r_{1} b+2 r_{2} a\right) c+r_{2} b^{2}+\left(2 r_{3}+r_{1}^{2}\right) b+\left(2 r_{1} r_{2}-4 r_{4}\right) a+r_{2}^{2}\right) u^{2} v^{2} / 2 \\
& +\left(2 r_{1} c+r_{2} b+r_{3}\right) u v^{2}+r_{2} v^{2} \\
& +\left(6\left(c+r_{1}\right) f^{2}+\left(r_{2} e+4 b c+9 r_{1} b+6 r_{2} a\right) f\right. \\
& +\left(2 c^{2}-5 r_{1} c-4 r_{2} b-5 r_{3}+r_{1}^{2}\right) e+2 a c^{2}+\left(b^{2}-16 r_{1} a+10 r_{2}\right) c \\
& \left.+3 r_{1} b^{2}-6 r_{2} a b+\left(2 r_{1}^{2}-10 r_{3}\right) a-4 r_{4}+14 r_{1} r_{2}\right) u^{3} v / 6 \\
& +\left(2\left(c+r_{1}\right) f-r_{2} e+b c+2 r_{1} b-2 r_{2} a\right) u^{2} v / 2+\left(c+r_{1}\right) u v \\
& +\left(6 f^{3}+11 b f^{2}+\left(\left(-3 c-10 r_{1}\right) e-12 a c+6 b^{2}-12 r_{1} a+6 r_{2}\right) f\right. \\
& +\left(-3 b c-10 r_{1} b+6 r_{2} a\right) e+20 c^{2}+4\left(7 r_{1}-a b\right) c+b^{3} \\
& \left.-2\left(8 r_{1} a+7 r_{2}\right) b-14 r_{3}+32 r_{1}^{2}\right) u^{4} / 24 \\
& +\left(2 f^{2}+3 b f-2 r_{1} e-c(e+4 a)+b^{2}-4 r_{1} a-2 r_{2}\right) u^{3} / 6 \\
& +(f+b) u^{2} / 2+u \\
y= & r_{5} v^{4}+\left(\left(2 r_{3}+r_{1}^{2}\right) f+\left(r_{4}+r_{1} r_{2}\right) e+r_{2}^{2}\right) u v^{3} \\
& +r_{3} v^{3}+\left(6 r_{1} f^{2}+3 r_{2} e f+\left(3 r_{1} c+r_{2} b-r_{3}+r_{1}^{2}\right) e\right. \\
& \left.\quad+2 r_{2} c-6 r_{3} a+2 r_{4}+2 r_{1} r_{2}\right) u^{2} v^{2} / 2 \\
& +\left(2 r_{1} f+r_{2} e\right) u v^{2}+r_{1} v^{2} \\
& +\left(6 f^{3}+\left(\left(6 c-r_{1}\right) e-24 r_{1} a+6 r_{2}\right) f\right. \\
& \quad-3 r_{2} e^{2}+\left(b c+r_{1} b-12 r_{2} a\right) e+2 c^{2} \\
& +\left(-a f+c r_{1} c+6 r_{2} b-2 r_{3}+14 r_{1}^{2}\right) u^{3} v / 6 \\
& +\left(2 f^{2}+c e-4 r_{1} a+2 r_{2}\right) u^{2} v / 2+f u v+v \\
& -\left(11 a f^{2}-12\left(c+r_{1}\right) f+\left(8 a c+4 r_{1} a+6 r_{2}\right) e-2\left(3 b+2 a^{2}\right) c\right. \\
& \left.+a b^{2}-12 r_{1} b-16 r_{1} a^{2}+4 r_{2} a\right) u^{4} / 12 \\
& \\
& \\
& \\
& \\
& \\
& \\
& \\
&
\end{aligned}
$$


For the normal form, we get

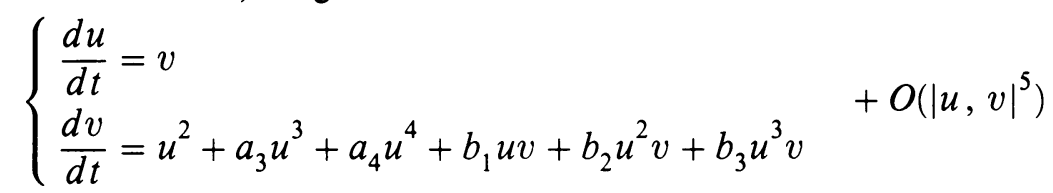

where $b_{1}=e+2 a, b_{2}$ depends on a parameter of the family in such a way that we may assume that $b_{2}=0$ without loss of generality. This choice once made: $b_{3}=-(b+2 f)\left(a f-c-a b-2 a^{3}\right), a_{3}, a_{4}$ are polynomials in $a, b, c, e, f$ and the parameters $r_{1} \cdots r_{6}$ of the family of transformations.

Assuming $b_{2}=0$, one easily checks that (9) is $C^{\infty}$-equivalent to

$$
\left\{\begin{array}{l}
\frac{d u}{d t}=v \\
\frac{d v}{d t}=u^{2}+b_{1} u v+3 \alpha b_{1} u^{2} v+\left(2\left(2 \beta+\alpha^{2}\right) b_{1}+b_{3}\right) u^{3} v
\end{array}+O\left(|u, v|^{5}\right)\right.
$$

where $\alpha=-a_{3} / 4$ and $\beta=\left(15 a_{3}^{2}-16 a_{4}\right) / 80$.

From this and [DRS1] the first two statements follow immediately. To have a cusp of codimension $>3$ at the origin, one of the following conditions $(\mathrm{C} 1)$ or (C2) must hold:

$$
(\mathrm{C} 1):\left\{\begin{array}{l}
e=-2 a \\
b=-2 f
\end{array} \quad(\mathrm{C} 2):\left\{\begin{array}{l}
e=-2 a \\
c=a\left(f-b-2 a^{2}\right)
\end{array}\right.\right.
$$

Under conditions $(\mathrm{C} 1)$, system $(6)$ is Hamiltonian. Suppose now that conditions (C2) are fullfilled. Making use of the linear coordinate change $x=X+a Y, y=$ $Y$ system $(6)$ is transformed into

$$
\left\{\begin{array}{l}
\frac{d X}{d t}=Y+\left(b+2 a^{2}\right) X Y \\
\frac{d Y}{d t}=X^{2}+\left(f-a^{2}\right) Y^{2}
\end{array}\right.
$$

System (11) is invariant under the transformation $(X, Y, t) \rightarrow(X,-Y,-t)$; and consequently it is symmetric with respect to the $X$-axis. Concerning the codimension, we refer to the normal form theory as presented in [Br]. A singularity with $y \frac{\partial}{\partial x}$ as 1 -jet, can be transformed by a $C^{\infty}$-diffeomorphism $\Phi$ into one with a formal normal form

$$
\left\{\begin{array}{l}
\frac{d u}{d t}=v+\sum_{n \geq 2} c_{n} u^{n} \\
\frac{d v}{d t}=\sum_{n \geq 2} a_{n} u^{n}
\end{array}\right.
$$

If such a singularity belongs to some Lie-algebra, like the Lie-algebra of Hamiltonian vector fields or the Lie-algebra $\left\{X \mid \Psi_{*} X= \pm X\right\}$ (with $\Psi(x, y)=$ $(x,-y))$, then $\Phi$ can be chosen in a way that the normal form belongs to the same Lie-algebra. In that way, in both cases, all $c_{n}$ in (12) need to be zero. This is the same as saying that all $b_{n}$ in (7) are zero, and it is clearly a situation of infinite codimension. 
Next we treat the case $d=0$.

Here also it is easy to find the family of near-identity transformations which convert system (6) into the normal form (7) up to terms of order 4 . The family is

$$
\left\{\begin{aligned}
x= & r_{6} v^{4}+\left(\left(2 r_{3}+r_{1}^{2}\right) c+\left(r_{4}+r_{1} r_{2}\right) b+r_{2}^{2} a+r_{5}\right) u v^{3}+r_{4} v^{3} \\
& +\left(\left(6 r_{1} c+r_{2} b+2 r_{3}+r_{1}^{2}\right) f+\left(2 r_{2} c-2 r_{4}+r_{1} r_{2}\right) e\right. \\
& \left.+\left(3 r_{1} b+2 r_{2} a\right) c+r_{2} b^{2}+\left(2 r_{3}+r_{1}^{2}\right) b+\left(2 r_{1} r_{2}-4 r_{4}\right) a\right) u^{2} v^{2} / 2 \\
& +\left(2 r_{1} c+r_{2} b+r_{3}\right) u v^{2}+r_{2} v^{2}+\left(6\left(c+r_{1}\right) f^{2}+\left(r_{2} e+4 b c+9 r_{1} b+6 r_{2} a\right) f\right. \\
& +\left(2 c^{2}-5 r_{1} c-4 r_{2} b-5 r_{3}+r_{1}^{2}\right) e+2 a c^{2}+\left(b^{2}-16 r_{1} a\right) c \\
& \left.+3 r_{1} b^{2}-6 r_{2} a b+\left(2 r_{1}^{2}-10 r_{3}\right) a\right) u^{3} v / 6 \\
& +\left(2\left(c+r_{1}\right) f-r_{2} e+b c+2 r_{1} b-2 r_{2} a\right) u^{2} v / 2+\left(c+r_{1}\right) u v \\
& +\left(6 f^{3}+11 b f^{2}+\left(\left(-3 c-10 r_{1}\right) e-12 a c+6 b^{2}-12 r_{1} a\right) f\right. \\
& \left.+\left(-3 b c-10 r_{1} b+6 r_{2} a\right) e-4 a b c+b^{3}-16 r_{1} a b\right) u^{4} / 24 \\
& +\left(2 f^{2}+3 b f-2 r_{1} e-c(e+4 a)+b^{2}-4 r_{1} a\right) u^{3} / 6 \\
& +(f+b) u^{2} / 2+u \\
y= & r_{5} v^{4}+\left(\left(2 r_{3}+r_{1}^{2}\right) f+\left(r_{4}+r_{1} r_{2}\right) e\right) u v^{3}+r_{3} v^{3} \\
& +\left(6 r_{1} f^{2}+3 r_{2} e f+\left(3 r_{1} c+r_{2} b-r_{3}+r_{1}^{2}\right) e-6 r_{3} a\right) u^{2} v^{2} / 2 \\
& +\left(2 r_{1} f+r_{2} e\right) u v^{2}+r_{1} v^{2} \\
& +\left(6 f^{3}+\left(\left(6 c-r_{1}\right) e-24 r_{1} a\right) f-3 r_{2} e^{2}+\left(b c+r_{1} b-12 r_{2} a\right) e\right) u^{3} v / 6 \\
& +\left(2 f^{2}+c e-4 r_{1} a\right) u^{2} v / 2+f u v+v \\
& +\left(11 a f^{2}+4 a\left(2 c+r_{1}\right) e-4 a^{2} c+a b^{2}-16 r_{1} a^{2}\right) u^{4} / 12-a f u^{3}-a u^{2}
\end{aligned}\right.
$$

The normal form reads

$$
\left\{\begin{array}{l}
\frac{d u}{d t}=v \\
\frac{d v}{d t}=a_{3} u^{3}+a_{4} u^{4}+b_{2} u v+b_{3} u^{2} v+b_{4} u^{3} v
\end{array}+O\left(|u, v|^{5}\right)\right.
$$

where

$$
\begin{aligned}
& a_{3}=-a e, \quad a_{4}=-a(f(e-2 a)+e b) / 2 \\
& b_{2}=e+2 a, \quad b_{3}=(f(e-2 a)+e b) / 2
\end{aligned}
$$

and $b_{4}$ is a polynomial in $a, b, c, e, f$ and the parameter $r_{1}$ of the family of transformations. Using (14), we can prove the next theorem:

Theorem 3. When $d=0$, the following statements can be made concerning the singularity at the origin in system (6): 
(1) If

$a e<0, \quad b \neq(2 a-e) f / e, \quad\left\{\begin{array}{ll}e \neq-2 a & \text { degenerate saddle of codimension } 3 \\ e=-2 a & \text { degenerate saddle of codimension } 4\end{array}\right.$.

(2) If

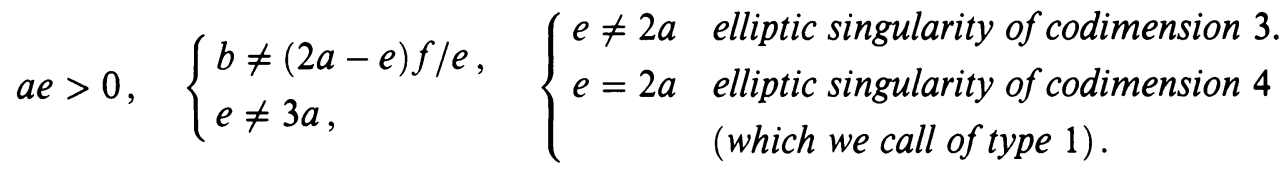

(3) If

$\begin{cases}e=3 a \neq 0, \quad 3 b+f \neq 0, & \text { elliptic singularity of codimension } 4 \\ (f-2 b)(3 f-b)+25 a c \neq 0, & \text { (which we call of type } 2) .\end{cases}$

(4) If

$\begin{cases}e=3 a \neq 0, \quad 3 b+f \neq 0, & \text { the singularity is approachable by } \\ (f-2 b)(3 f-b)+25 a c=0, & \text { centers, and hence of } \infty \text { codimension. }\end{cases}$

(5) If

the singularity is symmetric with respect

$a e \neq 0, \quad b=(2 a-e) f / e, \quad$ to an axis after a linear change

of coordinates, and hence of $\infty$ codimension.

(6) If

$a \neq 0, \quad e=0, \quad \begin{cases}f \neq 0, & \text { degenerate saddle-node of codimension } 4 . \\ f=0, & \text { a curve of singularities. }\end{cases}$

(7) If

$$
a=0, \quad \text { the } x \text {-axis is a line of singularities. }
$$

Proof. We prove (2) and (4), since the proof of the other statements is completely analogous to the proof of theorem 2 . One can show (see [DRS2]) that system (14) is $C^{\infty}$-equivalent to the system:

$$
\left\{\begin{aligned}
\frac{d x}{d t}= & y \\
\frac{d y}{d t}= & -a e x^{3}+y\left((e+2 a) x+\frac{(e-3 a)(f(e-2 a)+b e)}{5 e} x^{2}+O\left(x^{3}\right)\right) \\
& +y^{2} O\left(|x, y|^{2}\right)
\end{aligned}\right.
$$

After the rescaling $x=\alpha r, y=\beta s, t=\gamma \tau$, we get

$$
\left\{\begin{aligned}
\frac{d r}{d \tau}= & \frac{\beta \gamma}{\alpha} s \\
\frac{d s}{d \tau}= & \frac{-a e \alpha^{3} \gamma}{\beta} r^{3}+(e+2 a) \alpha \gamma r s+\frac{(e-3 a)(e b+(e-2 a) f) \alpha^{2} \gamma}{2} r^{2} s \\
& +s O\left(|r, s|^{3}\right)
\end{aligned}\right.
$$


We take $\alpha=\beta \gamma$. The coefficient of $r^{3}$ is then $-\left(a e \alpha^{3} \gamma\right) / \beta=-a e \beta^{2} \gamma^{4}$, and we impose $\beta^{2} \gamma^{4} a e=1$. Making the coefficient of $r^{2} s$ equal to \pm 1 has no effect on the coefficient of $r s$. For this coefficient we have $(e+2 a) \alpha \gamma=(e+2 a) \beta \gamma^{2}=$ $|e+2 a| / \sqrt{a e}$, if we choose $\operatorname{sign} \beta=\operatorname{sign}(e+2 a)$. Now

$$
(e-2 a)^{2} \geq 0 \Leftrightarrow e^{2}+4 a e+4 a^{2} \geq 8 a e \Leftrightarrow(e+2 a)^{2} \geq 8 a e \Leftrightarrow \frac{|e+2 a|}{\sqrt{a e}} \geq 2 \sqrt{2} .
$$

Conclusion: If $e \neq 2 a$ we have an elliptic singularity of codimension 3 , if $e=2 a$ we have an elliptic singularity of codimension 4 (of type 1). Here ends the proof of statement (2). Under the conditions of (4) and using linear coordinate changes and rescaling of time system (6) can be transformed into

$$
Z_{0}=\left(y+x^{2}+x y-2 y^{2} / 25\right) \frac{\partial}{\partial x}+3 x y \frac{\partial}{\partial y} .
$$

Let us introduce

$$
Z_{k}:\left\{\begin{array}{l}
\frac{d x}{d t}=y+\frac{25+4 k}{25} x^{2}+x y-\frac{2}{25} y^{2} \\
\frac{d y}{d t}=-k x+\frac{k}{5} x^{2}+\frac{75+2 k}{25} x y
\end{array}\right.
$$

For $k>0, Z_{k}$ has a center at the origin.

Remarks.

a. Concerning the nilpotent focus.

When $d=0$, the $x$-axis is an invariant line of system (6). Hence a degenerate nilpotent focus cannot exist in a quadratic system.

b. Concerning the saddle (resp. elliptic point) of codimension 4 .

We call it a saddle (resp. elliptic point) of codimension 4 since the normal form theorem together with the Tarski-Seidenberg decision theorem (see appendix [A.R.]) show that these singularities lay on a semialgebraic set of codimension 4. To our knowledge, there however does not exist a theorem showing that four parameters suffice to fully describe a versal unfolding of such a singularity. For the elliptic point, the distinction between type 1 and type 2 has to do with the value of certain coefficients in the normal form. We prefer not to give further specifications.

1.5. Homogeneous quadratic vector fields. We neglect the fact that these homogeneous quadratic vector fields are invariant under $(x, y, t) \mapsto(-x,-y,-t)$, which makes them presumably also of infinite codimension. However we miss a normal form theory in this case and therefore we like to check the behaviour of these singularities with respect to the properties which were valuable in the other cases.

Homogeneous quadratic vector fields always have an invariant line. We may suppose that after performing a rotation, we have an expression:

$$
X:\left\{\begin{array}{l}
\frac{d x}{d t}=a x^{2}+b x y+c y^{2} \\
\frac{d y}{d t}=e x y+f y^{2}
\end{array}\right.
$$


for which the $x$-axis is invariant. To avoid a line of singularities we may suppose that $a \neq 0$, which we do from now on. To study these singularities and especially their invariant lines, it is interesting to consider their polar blowing up (see [D]).

$$
\bar{X}:\left\{\begin{array}{l}
\frac{d \theta}{d t}=\sin \theta\left((e-a) \cos ^{2} \theta+(f-b) \sin \theta \cos \theta-c \sin ^{2} \theta\right) \\
\frac{d r}{d t}=r\left(a \cos ^{3} \theta+b \cos ^{2} \theta \sin \theta+(e+c) \cos \theta \sin ^{2} \theta+f \sin ^{3} \theta\right)
\end{array}\right.
$$

We will now distinguish different cases depending on the number of invariant straight lines through the origin.

1.5.1. $X$ has exactly 1 invariant line through the origin. This already implies that $c \neq 0$. There are now two possibilities:

(a)

$$
(f-b)^{2}+4 c(e-a)<0 .
$$

$\bar{X}$ has one symmetry pair of singularities $((0,0),(\pi, 0))$. The eigenvalues of $D \bar{X}((0,0))$ are respectively $e-a$ and $a$, which are both nonzero. Therefore $(0,0)$ and $(\pi, 0)$ are hyperbolic singularities for $\bar{X}$ and the singularities of $X$ may be considered of codimension 4. Presuming that the unfolding theory of these homogeneous singularities is not known, we will take the general codimension as to be the one defined in [D] for singularities.

(b)

$$
\begin{gathered}
f-b=0, \quad e-a=0, \quad c \neq 0, \\
\left\{\begin{array}{l}
\frac{d x}{d t}=a x^{2}+b x y+c y^{2}=(a x+b y) x+c y^{2} \\
\frac{d y}{d t}=a x y+b y^{2}=(a x+b y) y
\end{array}\right.
\end{gathered}
$$

We now consider the coordinate change $X=a x+b y, Y=y$ and get

$$
\left\{\begin{array}{l}
\frac{d X}{d t}=X^{2}+a c Y^{2} \\
\frac{d Y}{d t}=X Y
\end{array}\right.
$$

The system is symmetric with respect to the $Y$-axis, since it is invariant under $(X, Y, t) \mapsto(-X, Y,-t)$.

1.5.2. $X$ has at least two invariant lines through the origin. Up to a linear coordinate change, we may of course suppose that the $y$-axis is a second invariant line, meaning that $c=0$ in (16). To avoid a line of singularities we now suppose that $f \neq 0$. We end up with

$$
\left\{\begin{array}{l}
\frac{d x}{d t}=x(a x+b y) \\
\frac{d y}{d t}=y(e x+f y)
\end{array}\right.
$$


where $a, f \neq 0$. The polar expression is

$$
\left\{\begin{array}{l}
\frac{d \theta}{d t}=\sin \theta \cos \theta((e-a) \cos \theta+(f-b) \sin \theta) \\
\frac{d r}{d t}=r\left(a \cos ^{3} \theta+b \cos ^{2} \theta \sin \theta+e \cos \theta \sin ^{2} \theta+f \sin ^{3} \theta\right)
\end{array}\right.
$$

(a) $X$ has 3 invariant lines through the origin.

Necessarily $e \neq a$ and $f \neq b$ if we want to avoid a line of singularities. $\bar{X}$ having six singularities on $\{r=0\}$, the tangential eigenvalues of $D \bar{X}$ in these singularities are surely nonzero. Concerning the radial eigenvalues, we observe that they must be nonzero, since we keep the singularity of $X$ isolated. The six singularities of $\bar{X}$ are hence all hyperbolic, and in this sense we end up in a codimension 4-situation.

(b) $X$ has exactly two invariant lines through the origin.

The conditions are $(a \neq e$ and $b=f)$ or $(a=e$ and $b \neq f)$, and we obtain singularities of codimension 5. Up to switching $\mathrm{x}$ and $\mathrm{y}$, both cases reduce to the second one:

$$
\left\{\begin{array}{l}
\frac{d x}{d t}=x(a x+b y) \\
\frac{d y}{d t}=y(a x+f y)
\end{array}\right.
$$

with $b \neq f, a f \neq 0$. Multiplying with a nonzero factor, this reduces to:

$$
\left\{\begin{array}{l}
\frac{d x}{d t}=x(x+B y) \\
\frac{d y}{d t}=y(x+F y)
\end{array}\right.
$$

with $F \neq 0$ and $F \neq B$. After an extra coordinate change $(x, y) \rightarrow(x, y / F)$, we get:

$$
\left\{\begin{array}{l}
\frac{d x}{d t}=x(x+\beta y) \\
\frac{d y}{d t}=y(x+y)
\end{array}\right.
$$

with $\beta \neq 1$.

CHAPTER 2. QUADRATIC MODELS FOR GENERIC $k$-PARAMETER FAMILIES

$$
\text { WHEN } k \leq 3
$$

\subsection{Nilpotent case.}

2.1.1. Nilpotent cusps. Let $X_{0}$ be a germ of a vectorfield at $0 \in \mathbb{R}^{2}$ with $X_{0}(0)=0$ and with nilpotent 1 -jet, then by [T1] the 2-jet of $X_{0}$ is $C^{\infty}$ conjugate to

$$
y \frac{\partial}{\partial x}+\left(\alpha x^{2}+\beta x y\right) \frac{\partial}{\partial y}
$$


In generic 2-parameter families one may even suppose that $\alpha, \beta \neq 0$. In that case the 2 -jet is $C^{\infty}$-equivalent to

$$
y \frac{\partial}{\partial x}+\left(x^{2} \pm x y\right) \frac{\partial}{\partial y}
$$

and we say that the singularity is a cusp of codimension 2. In [B], Bogdanov has shown that all generic 2-parameter unfoldings of a cusp of codimension 2 are-up to time reversal-fiber- $C^{0}$-equivalent. For a definition of fiber- $C^{0}$ equivalence, we refer to [D2] or to [DRS1]. A simple model for this unfolding of codimension 2 is the well-known quadratic Bogdanov-Takens system

$$
y \frac{\partial}{\partial x}+\left(x^{2}+\mu+\nu y \pm x y\right) \frac{\partial}{\partial y} \text {. }
$$

In generic 3-parameter families, one locally encounters the cases $(\alpha \neq 0$ and $\beta=0)$ and $(\alpha=0$ and $\beta \neq 0)$.

First we treat the case $(\alpha \neq 0$ and $\beta=0)$.

To start we recall some results from [DRS1]. A singularity with nilpotent 1 -jet and whose 2-jet is $C^{\infty}$-equivalent to $y \frac{\partial}{\partial x}+\alpha x^{2} \frac{\partial}{\partial y}$ with $\alpha \neq 0$ is called a cusp singularity of codimension $\geq 3$. The set of germs of such vector fields constitute a semialgebraic subset of codimension 3 in the space of all germs of singular vector fields in $0 \in \mathbb{R}^{2}$. Denote this set by $\Sigma_{C}^{3}$. One can prove (see [DRS1]) that each $X_{0} \in \Sigma_{C}^{3}$ has a 4-jet $C^{\infty}$-equivalent to

$$
y \frac{\partial}{\partial x}+\left(x^{2}+\gamma x^{3} y\right) \frac{\partial}{\partial y}
$$

One defines $\Sigma_{C}^{4}$ by the condition $\gamma=0 ; \Sigma_{C}^{4}$ is a semialgebraic subset of codimension 1 in $\Sigma_{C}^{3}$ and $\Sigma_{C}^{3}=\Sigma_{C^{+}}^{3} \cup \Sigma_{C^{-}}^{3} \cup \Sigma_{C}^{4}$, where $\Sigma_{C^{ \pm}}^{3}$ is the submanifold of codimension 3 consisting of germs of singular vector fields whose 4-jet is $C^{\infty}$-equivalent to $y \frac{\partial}{\partial x}+\left(x^{2} \pm x^{3} y\right) \frac{\partial}{\partial y}$. The genericity condition of 3parameter families $X_{\lambda}$ with $X_{0} \in \Sigma_{C^{ \pm}}^{3}$ consists in the transversality of the mapping $(x, \lambda) \mapsto j^{4} X_{\lambda}(x)$ with respect to $\Sigma_{C^{ \pm}}^{3}$ in $(0,0)$. An example of such a family is given by

$$
\widetilde{X}_{\lambda}^{ \pm}=y \frac{\partial}{\partial x}+\left(x^{2}+\mu+y\left(\nu_{0}+\nu_{1} x \pm x^{3}\right)\right) \frac{\partial}{\partial y}
$$

with $\lambda=\left(\mu, \nu_{0}, \nu_{1}\right)$. The main result of [DRS1] can be stated as follows:

A local 3-parameter family in $(0,0) \in \mathbb{R}^{2} \times \mathbb{R}^{3}$ transversally cutting $\Sigma_{C^{ \pm}}^{3}$ at $(0,0)$ is fiber- $C^{0}$-equivalent to $\tilde{X}_{\lambda}^{+}$(resp. $\widetilde{X}_{\lambda}^{-}$).

Another result of [DRS1] is the following:

3-parameter families cutting $\Sigma_{C^{ \pm}}^{3}$ transversally can be brought-up to $C^{\infty}$. equivalence-in the following normal form:

(6) $X_{\lambda}=y \frac{\partial}{\partial x}+\left[x^{2}+\mu+y K(y, \lambda)\left(\nu_{0}+\nu_{1} x+\alpha(\lambda) x^{2} \pm x^{3}+x^{4} h(x, \lambda)\right)\right.$

$$
\left.+y^{2} Q(x, y, \lambda)\right] \frac{\partial}{\partial y}
$$


where $\lambda=\left(\mu, \nu_{0}, \nu_{1}\right), K(y, \lambda)$ is a $C^{\infty}$-function with $K(0, \lambda) \equiv 1, \alpha(\lambda)$ is a $C^{\infty}$-function in $\lambda$ with $\alpha(0)=0, h(x, \lambda)$ and $Q(x, y, \lambda)$ are $C^{\infty}$-functions and $Q$ is of order $\mathrm{N}$ in $(x, y, \lambda)$; where $\mathrm{N}$ is arbitrarily high, but given a priori. We will use this normal form to find a quadratic model of the unfolding of the cusp singularity of codimension 3. The procedure for our calculations consists of the next steps: First we search for a quadratic vector field $X_{0}$ such that $X_{0} \in \Sigma_{C^{+}}^{3}$. We start with the system

$$
\left\{\begin{array}{l}
\frac{d x}{d t}=y+P_{2}(x, y) \\
\frac{d y}{d t}=Q_{2}(x, y)
\end{array}\right.
$$

where $P_{2}(x, y)$ and $Q_{2}(x, y)$ are homogeneous polynomials of degree 2 . We know that this class of systems can be put in the normal form [T1, D, G.H.]

$$
\left\{\begin{array}{l}
\frac{d u}{d t}=v \\
\frac{d v}{d t}=\sum_{n \geq 2} a_{n} u^{n}+v \sum_{n \geq 2} b_{n} u^{n-1}
\end{array}\right.
$$

where $u$ and $v$ are related to $x$ and $y$ by a near-identity transformation. For these normal form calculations we use a Macsyma-program from R. Rand and D. Armbruster [R.A.]. So we calculate $a_{n}, b_{n}(n \leq 4)$ in function of the coefficients of the polynomials $P_{2}(x, y)$ and $Q_{2}(x, y)$ and we proceed by choosing values for these coefficients so that the 4-jet is already in normal form. In this way we find that the vector field

$$
X_{0}:\left\{\begin{array}{l}
\frac{d x}{d t}=y+x^{2}-2 x y-y^{2} / 2 \\
\frac{d y}{d t}=x^{2}-2 x y
\end{array}\right.
$$

is transformed into

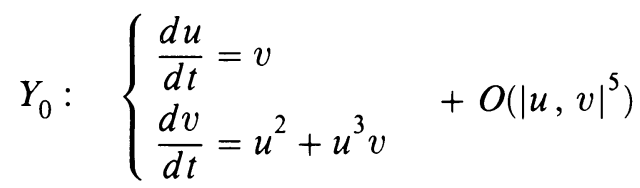

by the transformation

$$
\Phi:\left\{\begin{aligned}
x= & u-u^{2}+u v / 3+7 v^{2} / 10+3 u^{3} / 5-7 u^{2} v / 6-67 u v^{2} / 30 \\
& +317 u^{4} / 540+89 u^{3} v / 45+4591 u^{2} v^{2} / 1800-1843 u v^{3} / 1800 \\
y= & v-u^{2}+5 v^{2} / 6+u^{3} / 3-7 u^{2} v / 15-7 u v^{2} / 5 \\
& -u^{4} / 5+22 u^{3} v / 27+197 u^{2} v^{2} / 90-203 u v^{3} / 300
\end{aligned}\right.
$$

Then we apply the transformation to the vector field $X_{0}+X_{2}$ where $X_{2}=$ $\widetilde{P}_{2}(x, y) \frac{\partial}{\partial x}+\widetilde{Q}_{2}(x, y) \frac{\partial}{\partial y}$ with $\widetilde{P}_{2}(x, y)$ and $\widetilde{Q}_{2}(x, y)$ polynomials of degree 
2, not necessarily homogeneous. By choosing $\widetilde{P}_{2}(x, y)$ and $\widetilde{Q}_{2}(x, y)$ such that the 2-jet of $\Phi_{*}\left(X_{0}+X_{2}\right)$ is already in normal form (6), we find that the quadratic family

$$
X_{(a, b, c)}:\left\{\begin{aligned}
\frac{d x}{d t}= & a x / 3+(5+7 a) y / 5+(30+17 a) x^{2} / 30 \\
& +(15 b-206 a-90) x y / 45+(14(b-a)-5) y^{2} / 10 \\
\frac{d y}{d t}= & a+(5 a+3 b) y / 3+(5+6 a+5 b) x^{2} / 5 \\
& +(5 c-14 a-10) x y / 5+(15 b-25 a) y^{2} / 18
\end{aligned}\right.
$$

is transformed into

$$
Y_{(a, b, c)}:\left\{\begin{array}{l}
\frac{d u}{d t}=v \\
\frac{d v}{d t}=a+b v+u^{2}+c u v
\end{array} \quad+O\left(|u, v|^{3}\right)\right.
$$

(with $\left.Y_{(0,0,0)}=Y_{0}\right)$ by the transformation $\Phi$.

To conclude we prove that the quadratic family $X_{(a, b, c)}$ is indeed a versal unfolding of the cusp singularity of codimension 3. For this purpose it is not necessary to carry out the calculutions to put $Y_{(a, b, c)}$ in the normal form (6)up to terms of order 4 or higher-; it is sufficient to check that the parameters $a, b, c$ appear independent of each other in the 2-jet as in the normal form$a+b u+u^{2}+c u v-$ if we would carry out the calculations explicitly. One easily checks that the family $Y_{(a, b, c)}$ can be brought in normal form by means of transformations of the form

$$
\left\{\begin{array}{l}
u=x+T_{1}(x, y) \\
v=y+T_{2}(x, y)
\end{array}\right.
$$

where

$$
T_{l}(x, y)=\sum_{i, j, k \geq 0 ; i+j+k \geq 1} a^{i} b^{j} c^{k} \Phi_{i, j, k}^{l}(x, y)
$$

with

$$
\Phi_{i, j, k}^{l}(x, y)=O\left(|x, y|^{3}\right), \quad l=1,2
$$

and multiplication by a positive function $g(x, y)$. If we perform these calculations using the notation

$$
g(x, y)=1+g_{10} x+g_{01} y+g_{20} x^{2}+g_{11} x y+g_{02} y^{2}+O\left(|x, y|^{3}\right)
$$

and

$$
T_{l}(x, y)=\sum_{i, j \geq 0 ; i+j \geq 3} r_{l, i, j}(a, b, c) x^{i} y^{j}
$$


where $r_{l, i, j}(a, b, c)$ are polynomials in $a, b, c$ with $r_{l, i, j}(0,0,0)=0$, we find that $Y_{(a, b, c)}$ is transformed into

$$
Z_{(a, b, c)}:\left\{\begin{aligned}
\frac{d x}{d t}=y & -a r_{1,2,1}(a, b, c) x^{2} \\
& +\left(g_{10}-2 a r_{1,1,2}(a, b, c)\right) x y \\
& +\left(g_{01}-3 a r_{1,0,3}(a, b, c)\right) y^{2}+O\left(|x, y|^{3}\right) \\
\frac{d y}{d t}= & +a g_{10} x+\left(b+a g_{01}\right) y \\
+ & \left(1+a\left(g_{20}+r_{2,2,1}(a, b, c)\right)\right) x^{2} \\
+ & \left(c+a g_{11}+b g_{10}+2 a r_{2,1,2}(a, b, c)\right) x y \\
+ & \left(a g_{02}+b g_{01}+3 a r_{2,0,3}(a, b, c)\right) y^{2}+O\left(|x, y|^{3}\right)
\end{aligned}\right.
$$

So we directly see that the following conditions must hold:

$$
\begin{cases}r_{1,1,2}(a, b, c)=0, & g_{10}=0, \\ r_{1,2,1}(a, b, c)=0, & g_{01}=3 a r_{1,0,3}(a, b, c),\end{cases}
$$

and we get

$$
Z_{(a, b, c)}:\left\{\begin{aligned}
\frac{d x}{d t}=y & +O\left(|x, y|^{3}\right) \\
\frac{d y}{d t}= & a+\left(b+a g_{01}\right) y \\
& +\left(1+a\left(g_{20}+r_{2,2,1}(a, b, c)\right)\right) x^{2} \\
& +\left(c+a g_{11}+2 a r_{2,1,2}(a, b, c)\right) x y \\
& +\left(a g_{02}+b g_{01}+3 a r_{2,0,3}(a, b, c)\right) y^{2}+O\left(|x, y|^{3}\right)
\end{aligned}\right.
$$

In this way we proved that $X_{(a, b, c)}$ in (12) is a versal unfolding of the cusp of codimension 3 .

2.1.2. Nilpotent saddles and elliptic points. Now we study the other possibilities of codimension 3 when $(\alpha=0$ and $\beta \neq 0$ ). One can show that the germs of vector fields at $0 \in \mathbb{R}^{2}$ whose 1 -jet is nilpotent and whose 2 -jet is $C^{\infty}$-conjugate to $y \frac{\partial}{\partial x}+\left(\alpha x^{2}+\beta x y\right) \frac{\partial}{\partial y}$, with $\alpha=0$ and $\beta \neq 0$, have generically a 4-jet $C^{\infty}$ equivalent to

$$
y \frac{\partial}{\partial x}+\left(\varepsilon_{1} x^{3}+b x y+\varepsilon_{2} x^{2} y+d x^{3} y\right) \frac{\partial}{\partial y}
$$

with $b>0, \varepsilon_{1,2}= \pm 1, d \in \mathbb{R}$. It was shown in [D] that the topological type of such a germ is determined by its 3 -jet (if $b \neq 2 \sqrt{2}$ in case $\varepsilon_{1}=-1$ ). Actually it was proved that the topological type falls into one of the following classes:

(1) The saddle case: $\varepsilon_{1}=1$, any $b$ and $\varepsilon_{2}$ (a degenerate saddle) We denote by $\Sigma_{S^{ \pm}}^{3}\left(\varepsilon_{2}= \pm 1\right)$, the subsets of germs with such a 4 -jet. They all have the same topological type. 
(2) The elliptic case: $\varepsilon_{1}=-1$ and $b>2 \sqrt{2}$. We denote by $\Sigma_{E^{ \pm}}^{3}\left(\varepsilon_{2}= \pm 1\right)$ the corresponding subsets of germs. They all have the same topological type.

(3) The focus case: $\varepsilon_{1}=-1$ and $0<b<2 \sqrt{2}$. (a degenerate focus). Notation $\Sigma_{F^{ \pm}}^{3}\left(\varepsilon_{2}= \pm 1\right)$.

The article [DRS2] is devoted to the following conjecture:

Let $X_{\lambda}$ and $Y_{\lambda}$ be two local 3-parameter families, with $X_{0}$ and $Y_{0}$ belonging to the same set $\Sigma_{S^{ \pm}}^{3}, \Sigma_{E^{ \pm}}^{3}$ or $\Sigma_{F^{ \pm}}^{3}$. Suppose that both families are generic in the sense that the mapping $((x, y), \lambda) \in \mathbb{R}^{2} \times \mathbb{R}^{3} \mapsto j_{\lambda}^{4}((x, y))$ is transverse to the sets $\Sigma_{S^{ \pm}}^{3}, \Sigma_{E^{ \pm}}^{3}$ or $\Sigma_{F^{ \pm}}^{3}$ at $(0,0)$. Then they are fiber $C^{0}$-equivalent.

An example of such a generic family in each case is given by

$$
\tilde{X}_{\lambda}=y \frac{\partial}{\partial x}+\left(\varepsilon_{1} x^{3}+\mu_{2} x+\mu_{1}+y\left(\nu+b x+\varepsilon_{2} x^{2}\right)\right) \frac{\partial}{\partial y}
$$

where $\lambda=\left(\mu_{1}, \mu_{2}, \nu\right)$, and $b>0, b \neq 2 \sqrt{2}, \varepsilon_{1,2}= \pm 1$.

As in the cusp case one can prove that the 3-parameter families cutting $\Sigma_{S^{ \pm}}^{3}, \Sigma_{E^{ \pm}}^{3}$ or $\Sigma_{F^{ \pm}}^{3}$ transversally can be brought-up to $C^{\infty}$-equivalence-in a normal form. This normal form is

$\left.X_{\lambda}=y \frac{\partial}{\partial x}+\left(\varepsilon_{1} x^{3}+\mu_{2} x+\mu_{1}+y\left(\nu+b(\lambda) x+\varepsilon_{2} x^{2}\right)+x^{3} h(x, \lambda)\right)+y^{2} Q(x, y, \lambda)\right) \frac{\partial}{\partial y}$ where $\varepsilon_{1,2}= \pm 1, \lambda=\left(\mu_{1}, \mu_{2}, \nu\right)$ is the parameter, $b(\lambda)(b(0)>0)$ is a $C^{\infty}$-function and $Q$ is a $C^{\infty}$-function of order $N$ in $(x, y, \lambda)$, where $N$ is arbitrarily high, but given a priori.

First we treat the case of a quadratic unfolding of the degenerate saddle of codimension 3. Following a same procedure as in the cusp case, we find that the quadratic family

$$
S_{(a, b, c)}:\left\{\begin{array}{l}
\frac{d x}{d t}=y+x^{2}-2 x y \\
\frac{d y}{d t}=a+b x+c y+(b+c) x^{2}-x y
\end{array}\right.
$$

is transformed into

$$
\widetilde{S}_{(a, b, c)}:\left\{\begin{array}{l}
\frac{d u}{d t}=v \\
\frac{d v}{d t}=a+b u+c v+u v+(3-4 b-6 c) u^{3} / 3+u^{2} v
\end{array}+O\left(|u, v|^{4}\right)\right.
$$

by the near-identity transformation

$$
\left\{\begin{array}{l}
x=u-u^{2}+2 u^{3} / 3 \\
y=v-u^{2}
\end{array}\right.
$$

It is easy to check that $S_{(a, b, c)}$ is a versal unfolding of the degenerate saddle of codimension 3. A similar calculation will be performed in a more explicit way in Chapter 3. 
Next we treat the case of an elliptic singularity of codimension 3 . In the same way as before, we find that the quadratic family

$$
E_{(a, b, c)}:\left\{\begin{array}{l}
\frac{d x}{d t}=y+x^{2} / 2+x y \\
\frac{d y}{d t}=a+b x+c y+(c-b) x^{2} / 2+2 x y
\end{array}\right.
$$

is transformed into

$$
\widetilde{E}_{(a, b, c)}:\left\{\begin{array}{l}
\frac{d u}{d t}=v \\
\frac{d v}{d t}=a+b u+c v+3 u v-(6+2 b-3 c) u^{3} / 6+u^{2} v
\end{array}+O\left(|u, v|^{4}\right)\right.
$$

by the near-identity transformation

$$
\left\{\begin{array}{l}
x=u+u^{2} / 2+u^{3} / 6 \\
y=v-u^{2} / 2
\end{array}\right.
$$

Again one easily checks that $E_{(a, b, c)}$ will be a versal unfolding of the elliptic singularity of codimension 3 , if the conjecture made in [DRS2] concerning the elliptic point will get a positive answer.

We do not need to look at the focus case, since we observed in Chapter 1 that the nilpotent focus does not occur as a quadratic singularity.

2.1.3 Summary of 2.1 concerning generic unfoldings of codimension 3. To summarize the results of this section, let us list the three examples of 3-parameter bifurcations of codimension three, saying some words about their bifurcation diagram and showing also what it implies with respect to a question raised by Coppel in [C].

Theorem 4. The following quadratic families $X_{(a, b, c)}, S_{(a, b, c)}, E_{(a, b, c)}$ represent respectively:

(1) A nilpotent cusp bifurcation of codimension 3:

$$
X_{(a, b, c)}:\left\{\begin{aligned}
\frac{d x}{d t}= & a x / 3+(5+7 a) y / 5+(30+17 a) x^{2} / 30 \\
& +(15 b-206 a-90) x y / 45+(14(b-a)-5) y^{2} / 10 \\
\frac{d y}{d t}= & +(5 a+3 b) y / 3+(5+6 a+5 b) x^{2} / 5 \\
& +(5 c-14 a-10) x y / 5+(15 b-25 a) y^{2} / 18
\end{aligned}\right.
$$

(2) A nilpotent saddle bifurcation of codimension 3:

$$
S_{(a, b, c)}:\left\{\begin{array}{l}
\frac{d x}{d t}=y+x^{2}-2 x y \\
\frac{d y}{d t}=a+b x+c y+(b+c) x^{2}-x y
\end{array}\right.
$$




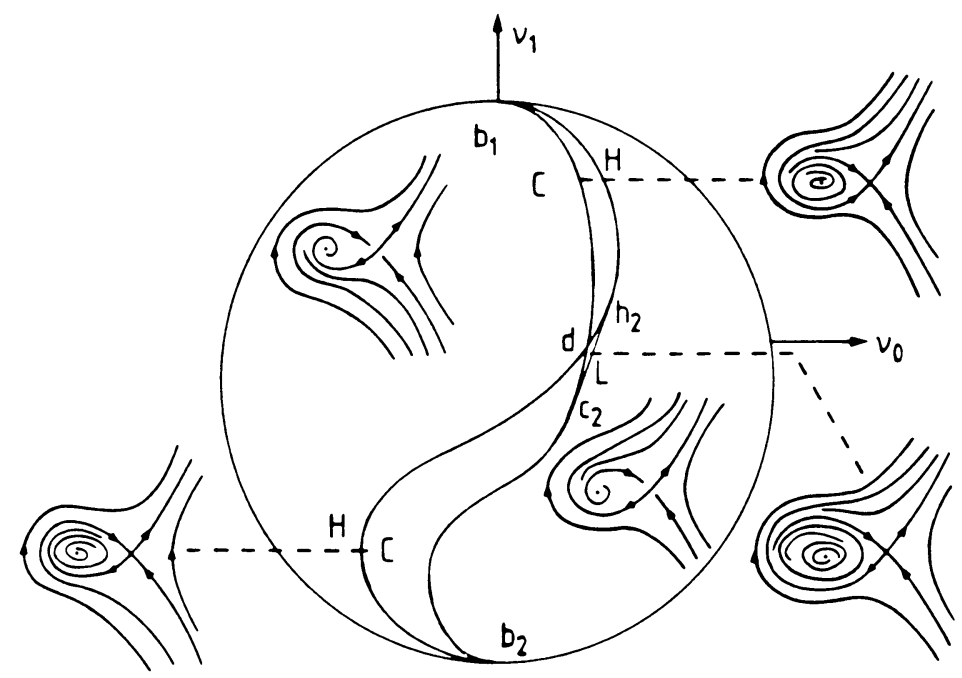

FIGURE 1. Bifurcation diagram and structurally stable phase portraits for the cusp case

(3) A nilpotent elliptic point bifurcation of codimension 3:

$$
E_{(a, b, c)}:\left\{\begin{array}{l}
\frac{d x}{d t}=y+x^{2} / 2+2 x y \\
\frac{d y}{d t}=a+b x+c y+(c-b) x^{2} / 2+2 x y
\end{array}\right.
$$

In each case the bifurcation diagram (in $(a, b, c)$-space) is a cone with respect to the origin. The intersection with small spheres can be drawn on a plane, using stereographic projection from a point corresponding to a parameter value for which the phase portrait is rather simple and structurally stable. These diagrams come from [DRS1] for the cusp case, and from [DRS2] for the saddle case. As the bifurcation diagram for the elliptic point is still conjectural we prefer not to draw it here.

Remarks. 1. We may observe that $X_{(a, b, c)}$ is an example of a family of quadratic systems showing for certain parameter values two limit cycles, which disappear (or appear) in a generic double cycle $(L)$, in a Hopf bifurcation of codimension $2\left(h_{2}\right)$, in a homoclinic loop of codimension $2\left(c_{2}\right)$ or in the simultaneous occurrence of a Hopf bifurcation (of codimension 1) $(H)$ and a homoclinic loop (of codimension 1) $(C)$. This phenomenon was also observed in $[R]$.

2. As the contact in $c_{2}$ between the line of double cycles $L$ and the line of homoclinic loop bifurcations $C$ is a flat contact (see [DRS1]) we know that the germ of this bifurcation diagram at the origin is not $C^{\infty}$-diffeomorphic to the 


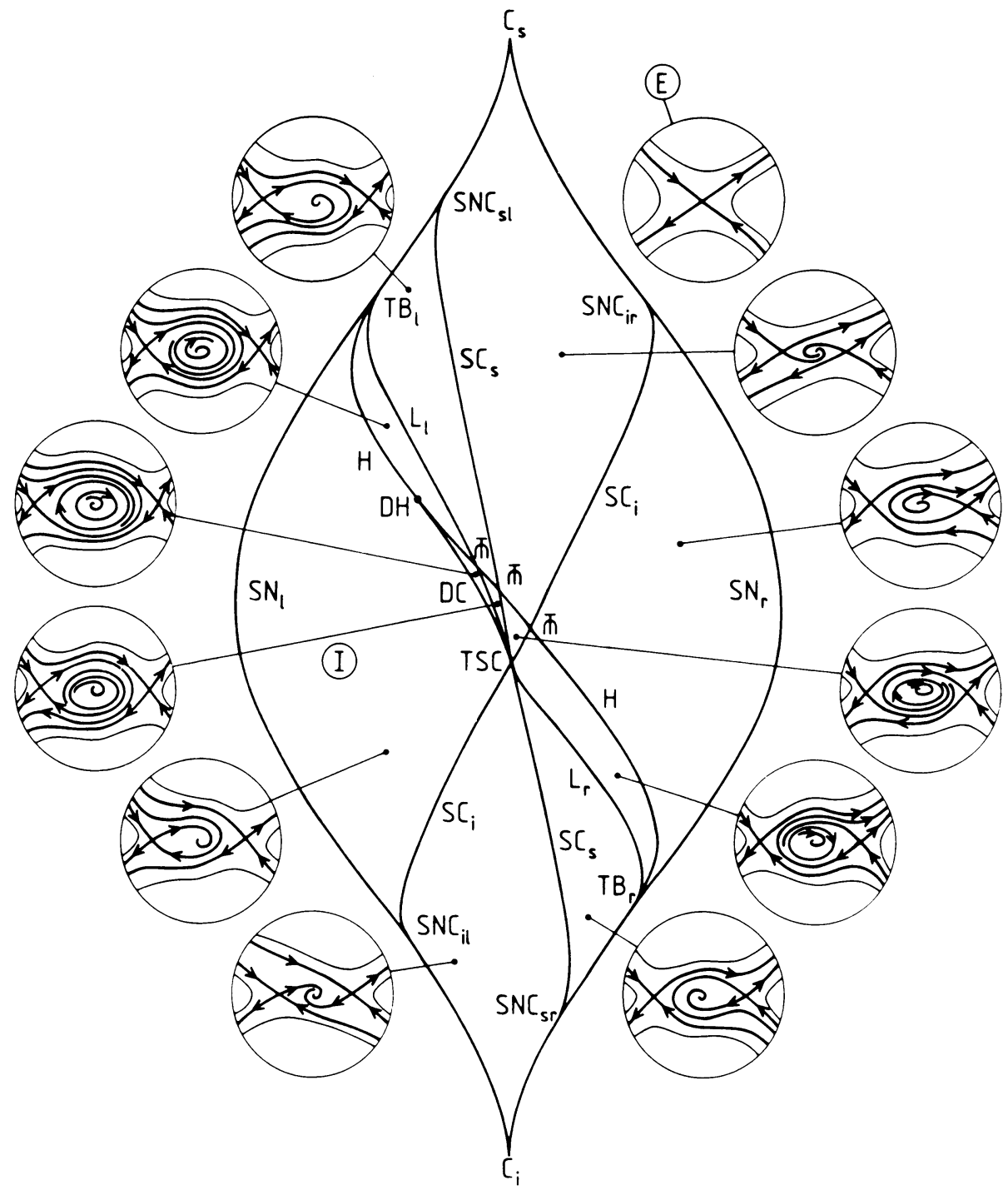

FigURE 2. Bifurcation diagram and structurally stable phase portraits for the saddle case

germ of an algebraic variety, even not to the germ of an analytic variety. This provides a negative answer to the question raised by Coppel in [C], at the end of his paper, concerning the algebraic characterization of the phase portraits of quadratic systems.

2.2. Semihyperbolic bifurcations and Hopf-Takens bifurcations. For the sake of completeness we add now quadratic models of local $k$-parameter families $(k \leq$ 3 ) representing versal unfoldings of semihyperbolic singularities (sometimes called generalized saddle-node bifurcations ) and representing Hopf-Takens bifurcations of codimension 1, 2, 3. As is well known the bifurcation diagrams 
for the semihyperbolic bifurcations only deal with singularities and are given by catastrophy-theory (resp. the fold-, the cusp-, and the swallowtail catastrophy). The bifurcation diagrams for the Hopf-Takens bifurcations can be found in [T2]; they only deal with the number of limit cycles around a unique singular point of focus type (nondegenerate or fine).

2.2.1. Semihyperbolic bifurcations. We proceed by recalling that an unfolding of a semihyperbolic singularity of codimension $k$ can be brought-up to $C^{\infty}$. equivalence-in the normal form:

$$
\begin{aligned}
\left(\mu_{0}+\mu_{1} x+\cdots+\mu_{k-1} x^{k-1}+\mu_{k} x^{k} \pm x^{k+1}\right) \frac{\partial}{\partial x} \\
\quad \pm y \frac{\partial}{\partial y}+O\left(|x, y|^{k+2}\right)+O\left(|\lambda|^{2}\right)
\end{aligned}
$$

The $\mu_{i}$ depend on the parameters $\left(\lambda_{1}, \ldots, \lambda_{k}\right)$ of the family in consideration and are all zero at the origin. It suffices to show that the mapping given by $\left(\mu_{0}, \ldots, \mu_{k-1}\right)$ has maximal rank at the origin. The standard model to which all codimension $k$ semihyperbolic bifurcations can be reduced to by $C^{0}$-equivalence is

$$
X_{k}^{ \pm, \pm}=\left(\nu_{0}+\nu_{1} x+\cdots+\nu_{k-1} x^{k-1} \pm x^{k+1}\right) \frac{\partial}{\partial x} \pm y \frac{\partial}{\partial y}
$$

where the $\left(\nu_{0}, \ldots, \nu_{k-1}\right)$ are independent parameters.

\section{Theorem 5.}

(1) The 1-parameter family

$$
S N_{(a)}:\left\{\begin{array}{l}
\frac{d x}{d t}=a+x^{2} \\
\frac{d y}{d t}=y
\end{array}\right.
$$

is a versal saddle-node bifurcation of codimension 1 .

(2) The 2-parameter family

$$
S N_{(a, b)}:\left\{\begin{array}{l}
\frac{d x}{d t}=a+b x+a y-a x^{2}+x y \\
\frac{d y}{d t}=2 a x+y+(2 b-1) x^{2}-2 a x y+y^{2}
\end{array}\right.
$$

is a versal semihyperbolic bifurcation of codimension 2 .

(3) The 3-parameter family

$$
S N_{(a, b, c)}:\left\{\begin{aligned}
\frac{d x}{d t}= & a+(b-2 a) x+(c-b-4 a) x^{2} \\
& -4 a x y+(2+2 a-b) y^{2} / 2 \\
\frac{d y}{d t}= & -2 a x+y+(1+4 a-2 b) x^{2}+(1+2 a) y^{2}
\end{aligned}\right.
$$

is a versal semihyperbolic bifurcation of codimension 3 . 
Proof. (2) The family $S N_{(a, b)}$ is transformed into

$$
\widetilde{S N}_{(a, b)}:\left\{\begin{array}{l}
\frac{d u}{d t}=a+b u+u^{3}-2 a u^{2} v+a v^{3} \\
\frac{d v}{d t}=v-2 a u^{3}+4 b u^{2} v-2 a u v^{2}
\end{array}+O\left(|u, v|^{4}\right)\right.
$$

by the near-identity transformation

$$
\left\{\begin{array}{l}
x=u+u v+u v^{2} \\
y=v+u^{2}+v^{2}+v^{3}
\end{array}\right.
$$

(3) The family $S N_{(a, b, c)}$ is $C^{\infty}$-equivalent to

$$
\widetilde{S N}_{(a, b, c)}:\left\{\begin{array}{l}
\frac{d u}{d t}=a+b u+c u^{2} \\
\frac{d v}{d t}=v
\end{array}+O\left(|u, v|^{3}\right)\right.
$$

with

$$
\widetilde{S N}_{(o, o, o)}:\left\{\begin{array}{l}
\frac{d u}{d t}=u^{4} \\
\frac{d v}{d t}=v
\end{array}+O\left(|u, v|^{5}\right)\right.
$$

by using the near-identity transformation

$$
\left\{\begin{array}{l}
x=u-u^{2}+v^{2} / 2-2 u^{2} v+2 v^{3} / 3+4 u^{3} v+2 u v^{3} / 3+3 v^{4} / 4 \\
y=v-u^{2}+v^{2}+2 u^{3}+u v^{2}+v^{3}-2 u^{4}+u^{2} v^{2}+5 u v^{3} / 3+13 v^{4} / 12
\end{array}\right.
$$

and multiplication with the function $g(u, v)=1+2 u^{2}$

2.2.2. Hopf-Takens bifurcations. We recall (see [T2]) that a Hopf-Takens bifurcation of codimension $k$ can be brought-up to $C^{\infty}$-equivalence-in the normal form

$$
\begin{aligned}
x \frac{\partial}{\partial y}-y \frac{\partial}{\partial x}-( & \left(x^{2}+y^{2}\right)^{k}+\mu_{1}\left(x^{2}+y^{2}\right)^{k-1} \\
& \left.+\cdots+\mu_{k-1}\left(x^{2}+y^{2}\right)+\mu_{k}\right)\left(x \frac{\partial}{\partial x}+y \frac{\partial}{\partial y}\right)+O\left(|x, y|^{2 k+12}\right)
\end{aligned}
$$

The $\mu_{i}$ depend on the parameters $\left(\lambda_{1}, \ldots, \lambda_{k}\right)$ of the family in consideration and are all zero at the origin. We need to show that the mapping given by $\left(\mu_{1}, \ldots, \mu_{k}\right.$ has maximal rank there. The standard model to which all codimension $k$ Hopf-Takens bifurcations can be reduced to by weak- $C^{\infty}$-equivalence (for a definition see [T2]) is

$$
\begin{aligned}
X_{k}^{ \pm}=x \frac{\partial}{\partial y}-y \frac{\partial}{\partial x}-\left( \pm\left(x^{2}+y^{2}\right)^{k}\right. & +\nu_{1}\left(x^{2}+y^{2}\right)^{k-1} \\
& \left.+\cdots+\nu_{k-1}\left(x^{2}+y^{2}\right)+\nu_{k}\right)\left(x \frac{\partial}{\partial x}+y \frac{\partial}{\partial y}\right)
\end{aligned}
$$

where the $\left(\nu_{1}, \ldots, \nu_{k}\right)$ are independent parameters. 


\section{Theorem 6.}

(1) The 1-parameter family

$$
H T_{(a)}:\left\{\begin{array}{l}
\frac{d x}{d t}=a x-y+(12+2 a) x^{2} / 3+(8 a-6) x y / 3-2 a y^{2} / 3 \\
\frac{d y}{d t}=x+a y+4 a x^{2} / 3+2 a x y / 3+8 a y^{2} / 3
\end{array}\right.
$$

is a versal Hopf-Takens bifurcation of codimension 1.

(2) The 2-parameter family

$$
H T_{(a, b)}:\left\{\begin{array}{l}
\frac{d x}{d t}=a x-y-x^{2}+(1+b) y^{2} \\
\frac{d y}{d t}=x+a y+2 x y+2 y^{2}
\end{array}\right.
$$

is a versal Hopf-Takens bifurcation of codimension 2.

(3) The 3-parameter family

$$
H T_{(a, b, c)}:\left\{\begin{array}{l}
\frac{d x}{d t}=a x-y+x^{2}+(5+b) x y-y^{2} \\
\frac{d y}{d t}=x+a y+x^{2}+(c-2) x y
\end{array}\right.
$$

is a versal Hopf-Takens bifurcation of codimension 3.

Proof. The family $H T_{(a)}$ is transformed into

$$
\widetilde{H T}_{(a)}:\left\{\begin{array}{l}
\frac{d u}{d t}=a u-v \\
\frac{d v}{d t}=u+a v
\end{array}+O\left(|u, v|^{3}\right)\right.
$$

with

$$
\widetilde{H T}_{(0)}:\left\{\begin{array}{c}
\frac{d u}{d t}=-v-u\left(u^{2}+v^{2}\right) \\
\frac{d v}{d t}=u-v\left(u^{2}+v^{2}\right)
\end{array}+O\left(|u, v|^{4}\right)\right.
$$

by use of the near-identity transformation

$$
\left\{\begin{array}{l}
x=u+2 u^{2} / 3+8 u v / 3-2 v^{2} / 3-5 u^{3}+7 u^{2} v+3 u v^{2} / 2 \\
y=v+4 u^{2} / 3+2 u v / 3+8 v^{2} / 3-10 u^{3} / 3-13 u^{2} v / 6-u v^{2}
\end{array}\right.
$$

and multiplication with the function

$$
g(u, v)=1+\left(17 u^{2}+v^{2}\right) / 6
$$

The method of proof for (2) and (3) is completely similar. We prefer not to include it since the expressions are too long. 


\section{Chapter 3. ON THE VERSAL UNFOlding OF THE ElLiptic POINT OF CODIMENSION 4 AND TYPE 1}

In this section we will consider all elliptic points of codimension 4 and type 1 as obtained in Theorem 3 and we will show that such a singularity can never have a quadratic versal unfolding. This fact does not really need to be further argumented since we already know that a quadratic nilpotent focus does not occur, while near the elliptic point of codimension 4 (as a general system) one obtains nilpotent foci merely by making the coefficient before $r s \frac{\partial}{\partial s}$ in the normal form (14) (see Chapter 1) less than $2 \sqrt{2}$. Nevertheless we will make the necessary normal form calculations in order to show that this coefficient before $r s \frac{\partial}{\partial s}$ always undergoes a generic fold near such an elliptic point of codimension 4 and type 1 . As seen in Theorem 3 of Chapter 1 the expression of these quadratic vector fields is

$$
\left\{\begin{array}{l}
\frac{d x}{d t}=y+a x^{2}+b x y+c y^{2} \\
\frac{d y}{d t}=2 a x y+f y^{2}
\end{array}\right.
$$

with $a \neq 0, b \neq 0$. We may suppose $a>0$. Let us consider quadratic perturbations having at the origin a singularity with 1 -jet $y \frac{\partial}{\partial x}$ and which is not of cusp type.

$$
\left\{\begin{array}{l}
\frac{d x}{d t}=y+\left(a+a_{20}\right) x^{2}+\left(b+a_{11}\right) x y+\left(c+a_{02}\right) y^{2} \\
\frac{d y}{d t}=\left(2 a+b_{11}\right) x y+\left(f+b_{02}\right) y^{2}
\end{array}\right.
$$

We perform the coordinate change

$$
\left\{\begin{array}{l}
u=x \\
v=y+\left(a+a_{20}\right) x^{2}+\left(b+a_{11}\right) x y+\left(c+a_{02}\right) y^{2}
\end{array}\right.
$$

and get

$$
\left\{\begin{array}{l}
\frac{d u}{d t}=v \\
\frac{d v}{d t}=-c_{30} u^{3}+O\left(u^{4}\right)+v\left(c_{11} u+O\left(u^{2}\right)\right)+v^{2} O(1)
\end{array}\right.
$$

with

$$
\left\{\begin{array}{l}
c_{30}=2 a^{2}+a\left(b_{11}+2 a_{20}\right)+a_{20} b_{11} \\
c_{11}=4 a+b_{11}+2 a_{20}
\end{array}\right.
$$

Using Macsyma it is easy to show that system (4) is $C^{\infty}$-conjugate to

$$
\left\{\begin{array}{l}
\frac{d u}{d t}=v \\
\frac{d v}{d t}=-c_{30} u^{3}+O\left(u^{4}\right)+v\left(c_{11} u+O\left(u^{2}\right)\right)+v^{2} O\left(|u, v|^{2}\right)
\end{array}\right.
$$


From now on we work with the family of dual forms:

$$
v d v+\left(c_{30} u^{3}+O\left(u^{4}\right)\right) d u-v\left(c_{11} u+O\left(u^{2}\right)\right) d u-v^{2} O\left(|u, v|^{2}\right) d u
$$

There exists a transformation of the form

$$
\left\{\begin{array}{l}
r=c_{30}^{1 / 4} u+O\left(u^{2}\right) \\
s=v
\end{array}\right.
$$

which changes the family into

$$
s d s+r^{3} d r-s\left(\left(c_{11} / \sqrt{c}_{30}\right) r+O\left(r^{2}\right)\right) d r-s^{2} O\left(|r, s|^{2}\right) d r .
$$

Direct calculation shows that the coefficient of $-r s d r, c_{11} / \sqrt{c}_{30}$, is always greater than or equal to $2 \sqrt{2}$. Moreover, the Taylor expansion of this coefficient is given by

$$
2 \sqrt{2}+\frac{\sqrt{2}\left(b_{11}-2 a_{20}\right)^{2}}{16 a^{2}}+O\left(\left|b_{11}, a_{20}\right|^{3}\right) .
$$

So we see that the coefficient undergoes a fold. We end by proving the genericity of this fold. For this purpose we show that bringing the family

$$
X_{\lambda}:\left\{\begin{array}{l}
\frac{d x}{d t}=y+a x^{2}+b x y+c y^{2} \\
\frac{d y}{d t}=\lambda_{1}+\lambda_{2} x+\lambda_{3} y+2 a x y+f y^{2}
\end{array}\right.
$$

with $\lambda=\left(\lambda_{1}, \lambda_{2}, \lambda_{3}\right)$ into the normal form

(11) $s \frac{\partial}{\partial r}-\left[-r^{3}+\mu_{2}(\lambda) r+\mu_{1}(\lambda)+s\left(\nu(\lambda)+b(\lambda) r \pm r^{2}+r^{3} h(r, \lambda)\right)+s^{2} Q(r, s, \lambda)\right] \frac{\partial}{\partial s}$

the map $\mathbb{R}^{3} \rightarrow \mathbb{R}^{3}, \lambda \mapsto\left(\mu_{1}(\lambda), \mu_{2}(\lambda), \nu(\lambda)\right)$ satisfies the condition

$$
\frac{D\left(\mu_{1}, \mu_{2}, \nu\right)}{D\left(\lambda_{1}, \lambda_{2}, \lambda_{3}\right)}(0) \neq 0 \text {. }
$$

To compute this determinant we treat every parameter $\lambda_{j}, j=1,2,3$, separately. Consider the family

$$
\left\{\begin{array}{l}
\frac{d x}{d t}=y+a x^{2}+b x y+c y^{2} \\
\frac{d y}{d t}=\lambda_{3} y+2 a x y+f y^{2}
\end{array}\right.
$$

Using the coordinate transformation

$$
\left\{\begin{array}{l}
x=u+\frac{b+f}{2} u^{2}+c u v+\frac{\left(b^{2}-2 a c+3 b f+2 f^{2}-c \lambda_{3}\right)}{6} u^{3}+\frac{c(b+2 f)}{2} u^{2} v \\
y=v-a u^{2}+f u v-a f u^{3}+\frac{2 f^{2}-2 a c+c \lambda_{3}}{2} u^{2} v
\end{array}\right.
$$


one easily proofs that system (13) is $C^{\infty}$-conjugate to

$$
\left\{\begin{array}{l}
\frac{d u}{d t}=v \\
\frac{d v}{d t}=-a \lambda_{3} u^{2}-2 a^{2} u^{3}+O\left(u^{4}\right)+v\left(\lambda_{3}+4 a u+O\left(u^{2}\right)\right)+v^{2} O\left(|u, v|^{2}\right)
\end{array}\right.
$$

Now we consider the family of dual forms

(16) $v d v+\left(a \lambda_{3} u^{2}+2 a^{2} u^{3}+O\left(u^{4}\right)\right) d u-v\left(\lambda_{3}+4 a u+O\left(u^{2}\right)\right) d u-v^{2} O\left(|u, v|^{2}\right) d u$.

Denote $f\left(u, \lambda_{3}\right)=a \lambda_{3} u^{3} / 3+2 a^{2} u^{4} / 4+O\left(u^{5}\right)$. Using an adapted form of the preparation theorem [T2] we find a coordinate change of the form

$$
u=\Phi\left(U, \lambda_{3}\right)=\phi_{1}\left(\lambda_{3}\right) U+\phi_{2}\left(\lambda_{3}\right) U^{2}+O\left(U^{3}\right)
$$

with $\phi_{1}(0)=\left(2 a^{2}\right)^{-1 / 4}$ such that

$$
f\left(\Phi\left(U, \lambda_{3}\right), \lambda_{3}\right)=U^{4} / 4+\eta\left(\lambda_{3}\right) U^{3} / 3
$$

with

$$
\eta\left(\lambda_{3}\right)=\frac{a \lambda_{3}}{\left(2 a^{2}\right)^{3 / 4}}+O\left(\lambda_{3}^{2}\right) .
$$

Performing this coordinate transformation to the family (16) we get

(20) $v d v+\left(U^{3}+\eta\left(\lambda_{3}\right) U^{2}\right) d U$

$$
\begin{aligned}
-v\left(\lambda_{3} \phi_{1}\left(\lambda_{3}\right)+\left(2 \lambda_{3} \phi_{2}\left(\lambda_{3}\right)+4 a \phi_{1}\left(\lambda_{3}\right)^{2}\right) U+\right. & \left.O\left(U^{2}\right)\right) d U \\
& \left.-v^{2} O\left(|U, v|^{2}\right)\right) d U
\end{aligned}
$$

Further we use the translation

$$
\left\{\begin{array}{l}
U=r-\eta\left(\lambda_{3}\right) / 3 \\
v=s
\end{array}\right.
$$

and we obtain

$$
\begin{aligned}
& s d s+\left(r^{3}-\left(\eta\left(\lambda_{3}\right)\right)^{2} r / 3+2\left(\eta\left(\lambda_{3}\right)\right)^{3} / 27\right) d r \\
& -s\left(\lambda_{3} \phi_{1}\left(\lambda_{3}\right)-\left(2 \lambda_{3} \phi_{2}\left(\lambda_{3}\right)+4 a \phi_{1}\left(\lambda_{3}\right)^{2}\right) \eta\left(\lambda_{3}\right) / 3+O\left(\left(\lambda_{3}\right)^{2}\right)+O(r)\right) d r \\
& -s^{2} O\left(|r, s|^{2}\right) d r
\end{aligned}
$$

and we can conclude that $\frac{\partial \mu_{1}}{\partial \lambda_{3}}(0)=0, \frac{\partial \mu_{2}}{\partial \lambda_{3}}(0)=0$ and $\frac{\partial \nu}{\partial \lambda_{3}}(0) \neq 0$. Following a same procedure for $\lambda_{2}$ and $\lambda_{1}$ we find that condition (12) is satisfied.

\section{REFERENCES}

[A.R.] R. Abraham and J. Robbin, Transversal mappings and flows, Benjamin, New York, 1967.

[B] N. N. Bautin, On the number of limit cycles which appear with the variation of coefficients from an equilibrium position of focus or center type, Mat. Sb. 30(72) (1952), 181-196; English transl., Amer. Math. Soc. Transl. No 100 (1954). 
[Br] $\mathrm{H}$. Broer, Formal normal form theorems for vector fields and some consequences for bifurcations in the volume preserving case, Dyn. Syst. and Turbulence, Warwick, 1980, Lecture Notes in Math., vol. 898, Springer-Verlag, 1981, pp. 54-74.

[C] W. A. Coppel, A survey of quadratic systems, J. Differential Equations 2 (1966) 293-304.

[D1] F. Dumortier, Singularities of vector fields on the plane, J. Differential Equations 23 (1977), 53-106.

[D2] _- Singularities of vector fields, Monogr. Mat. 32, IMPA, Rio de Janeiro, 1978.

[DRS1] F. Dumortier, R. Roussarie, and J. Sotomayor, Generic 3-parameter families of vector fields on the plane, unfolding a singularity with nilpotent linear part. The cusp case, Ergodic Theory Dynamical Systems 7 (1987), 375-413.

[DRS2] F. Dumortier, R. Roussarie, and J. Sotomayor, Generic 3-parameter families of planar vector fields, unfoldings of saddle, focus and elliptic singularities with nilpotent linear parts, Lecture Notes in Math., Springer-Verlag (to appear).

[G.H.] J. Guckenheimer, P. Holmes, Non-linear oscillations, dynamical systems and bifurcations of vector fields, Appl. Math. Sci. 42, Springer-Verlag, 1983.

[R] C. Rousseau, Example of a quadratic system with two cycles appearing in a homoclinic loop bifurcation, J. Differential Equations 66 (1987), 140-150.

[R.A.] R. Rand and D. Armbruster, Perturbation methods, bifurcation theory and computer algebra, Appl. Math. Sci. 65, Springer-Verlag, 1987.

[S] D. Schlomiuk, Personal Communication.

[T1] F. Takens, Singularities of vector fields, Publ. Math. IHES 43 (1974), 47-100.

[T2] _ - Unfoldings of certain singularities of vector fields. Generalized Hopf bifurcations, J. Differential Equations 14 (1973), 476-493.

[Y] Ye Yanqian et al., Theory of limit cycles, Transl. Math. Monos., vol. 66, Amer. Math. Soc., Providence, R.I., 1986.

Limburgs Universitair Centrum, Universitaire Campus, B-3590 Diepenbeek, Belgium 